\title{
Volume-Targeted Versus Pressure-Targeted Noninvasive Ventilation in Patients With Chest-Wall Deformity: A Pilot Study
}

\author{
Fransien M Struik MSc, Marieke L Duiverman MD PhD, Petra M Meijer NP, \\ Jellie A Nieuwenhuis MD, Huib AM Kerstjens MD PhD, \\ and Peter J Wijkstra MD PhD
}

\begin{abstract}
BACKGROUND: Long-term noninvasive ventilation (NIV) is an effective treatment for patients with chronic respiratory failure due to chest-wall deformity, but it is unknown if the time required for the patient to adjust to long-term NIV depends on whether the NIV is volume-targeted or pressure-targeted. OBJECTIVE: To determine whether volume controlled or pressure controlled NIV is easier to implement in patients with chronic respiratory failure due to chest-wall deformity. METHODS: We randomized 16 ventilator-naïve patients to receive either volume-targeted or pressure-targeted nocturnal NIV. The primary outcome was the number of days needed to successfully establish NIV, defined as adequate adjustment and effective ventilation, as measured with overnight arterial blood gas measurement. RESULTS: Two patients did not tolerate volume NIV, and switched to pressure NIV. NIV was successfully established in both groups after a median 6.0 days. There were no significant differences between the groups at any time point in $\mathrm{PaCO}_{2}$ or $\mathrm{P}_{\mathrm{aO}}$ improvement, nor in changes over time. CONCLUSIONS: There was no significant difference in days needed to successfully establish volume NIV versus pressure NIV in patients with chest-wall deformity. However, two patients switched successfully from volume NIV to pressure NIV, which suggests that they preferred pressure NIV. Key words: noninvasive ventilation; NIV; blood gas analysis; chronic respiratory insufficiency. [Respir Care 2011;56(10):1522-1525. (C) 2011 Daedalus Enterprises]
\end{abstract}

\section{Introduction}

Patients with severe chest-wall deformity have a decreased capacity of the respiratory muscles due to rib-cage deformity, which can lead to chronic respiratory failure. Since several decades, long-term noninvasive ventilation (NIV) has become a standard treatment in patients with chronic respiratory failure due to chest-wall deformity. ${ }^{1}$ NIV can improve arterial blood gas levels, daytime symptoms of hypoventilation, and quality of sleep..$^{2-4}$ An observational study on nasal intermit-

The authors are affiliated with the Department of Pulmonary Diseases and Home Mechanical Ventilation, University Medical Center Groningen, University of Groningen, Groningen, The Netherlands.

The authors have disclosed no conflicts of interest.

Correspondence: Fransien M Struik MSc, Department of Pulmonary Diseases and Home Mechanical Ventilation, University Medical Center Groningen, University of Groningen, Hanzeplein 1, Post Box 30.001, 9700 RB Groningen, The Netherlands. E-mail: f.m.struik@umcg.nl.

DOI: $10.4187 /$ respcare.01043 tent NIV also found a reduction in hospital days for respiratory illness in patients with chest-wall deformity for more than 2 years while receiving nasal intermittent NIV. ${ }^{5}$

Since the 1980s, volume-targeted NIV has been the predominant type of NIV used in patients with chronic respiratory failure due to chest-wall deformity. In the last decade, however, pressure-targeted NIV has become a widely accepted alternative. In volume-targeted NIV a pre-set tidal volume is given, which leads to a fluctuating inspiratory pressure that depends on the airway resistance and the respiratory system compliance. Pressure-targeted NIV delivers a predetermined pressure that results in different tidal volumes. The findings of studies of volume versus pressure NIV have been contradictory. ${ }^{6,7}$ More recent studies found that both volumetargeted and pressure-targeted long-term NIV are equally effective in patients with chest-wall deformity, with regard to improvements in gas exchange, sleep quality, physical activity, and health-related quality of life,, 89 but those studies were either uncontrolled or included patients who were already instituted on NIV. Two recent studies looked at the institution of NIV in NIV-naïve patients, but the focus of those studies 


\section{Volume Versus Pressure NiV in Patients With Chest-Wall Deformity}

was more on the different modes (pure controlled NIV, assisted ventilation, or "average volume assured pressure support") than on volume NIV versus pressure NIV.10,11

Remarkably, even though existing recommendations state when to start NIV, ${ }^{1}$ there are no guidelines for the choice between volume NIV and pressure NIV in NIV-naïve patients. Assuming equal effectiveness of gas exchange, a difference in time to adequate patient adjustment to NIV could help us decide whether pressure NIV or volume NIV is the better choice. We hypothesized that in patients with chestwall deformity it would take fewer days to adjust to pressuretargeted NIV than to volume-targeted NIV.

\section{Methods}

The study was conducted in our out-patient respiratory clinic in the University Medical Center Groningen, The Netherlands, between 2003 and 2008. The study was approved by our ethics committee, and all patients gave informed consent.

\section{Subjects}

We screened patients who had chest-wall deformity, slowly progressive chronic respiratory failure $\left(\mathrm{P}_{\mathrm{aCO}}\right.$ $>45 \mathrm{~mm} \mathrm{Hg}$ ), and one of the following symptoms of nocturnal hypoventilation: daytime sleepiness, fatigue, morning headaches and/or dyspnea, or weight loss. All the subjects were NIV-naïve.

\section{Study Intervention}

Patients were randomized to receive either volume-targeted or pressure-targeted NIV. The randomization sequence was via lettered cards denoting volume or pressure NIV, which were placed in sealed unmarked envelopes. Both types of NIV were initiated in hospital, on a normal respiratory ward, with close supervision of medical and nursing staff. All patients underwent blood gas analysis during a night in the intensive care unit (ICU) before the day of NIV initiation, and on the day of discharge. For volume NIV we used the continuous mandatory ventilation mode on the Breas 501 ventilator (Breas Medical, Mölndal, Sweden). For pressure NIV we used the spontaneous/time-cycled mode on the Synchrony 1 (Respironics, Murrysville, Pennsylvania). Settings were adjusted to deliver the maximum calculated volume $(8-10 \mathrm{~mL} / \mathrm{kg})$ or the highest pressure the patient could tolerate. The aim of ventilation was to decrease $\mathrm{P}_{\mathrm{aCO}}$ to $<45 \mathrm{~mm} \mathrm{Hg}$, maintain $\mathrm{S}_{\mathrm{aO}_{2}}$ above $90 \%$, and improve symptoms while maintaining patient comfort. The patients were admitted to the ICU to measure overnight blood gasses, via arterial line, every 2 hours, and the ventilation settings were adjusted as necessary based on the arterial blood gas values. If the above-mentioned criteria were not achieved, the patient would stay a second night for additional ventilation-settings adjustments. All the patients started NIV with nasal mask but changed, if necessary, to oronasal mask. After 3 months the patients were readmitted to the ICU for overnight blood-gas monitoring. Arterial blood gas analysis was conducted before initiating NIV (6:00 AM, upon awakening), on the morning of discharge after the patient slept adequately on NIV (6:00 AM, upon awakening), and after 3 months of nocturnal NIV at home (6:00 AM, upon awakening).

\section{Study Outcome}

Our primary outcome was the number of days needed to successfully establish the patient on NIV, defined as adequate patient-adjustment to NIV and effective ventilation $\left(\mathrm{P}_{\mathrm{aCO}_{2}}<45 \mathrm{~mm} \mathrm{Hg}\right)$. NIV adjustment was considered adequate when the patient could sleep with the NIV for at least 6 hours per night.

\section{Statistical Analysis}

Data are presented as mean \pm SD or median \pm range. Differences between the pressure and volume NIV groups were determined with the 2-sided Mann-Whitney test. Differences within each group were determined with the 2 -sided Wilcoxon signed-rank test. A $P$ value $<.05$ was considered statistically significant.

\section{Results}

Sixteen patients were included: 9 started with volume NIV, and 7 started with pressure NIV. Two patients did not tolerate volume NIV and switched to pressure NIV after 2 days. We assessed those 2 patients as being included in the pressure group, starting the counting on the day they switched. This resulted in 8 patients in the pressure group and 5 in the volume group. Three patients dropped out: 2 died (one from cancer, the other from pneumothorax), and one did not want to return for measurements in hospital at 3 months, though that patient was still using NIV at home. Thirteen patients completed the study. Figure 1 shows the flow chart, which follows the Consort guidelines (http://www.consortstatement.org). Table 1 shows the cohort's baseline characteristics and mean initial NIV settings.

NIV was successfully established in the volume group and pressure group after a median of 6 days (range $4-8$ days in the volume group, 5-14 days in the pressure group). If we include the days on volume NIV in the 2 patients who switched to pressure NIV, NIV establishment in the pressure group took a median 7 days (range 5-14 days), which was not significantly different than the volume group.

Both groups showed $\mathrm{P}_{\mathrm{aCO}}$ improvement between the NIV initiation and day of discharge, and that change was maintained and significant in both groups at 3 months, but there was no significant difference between the groups, 


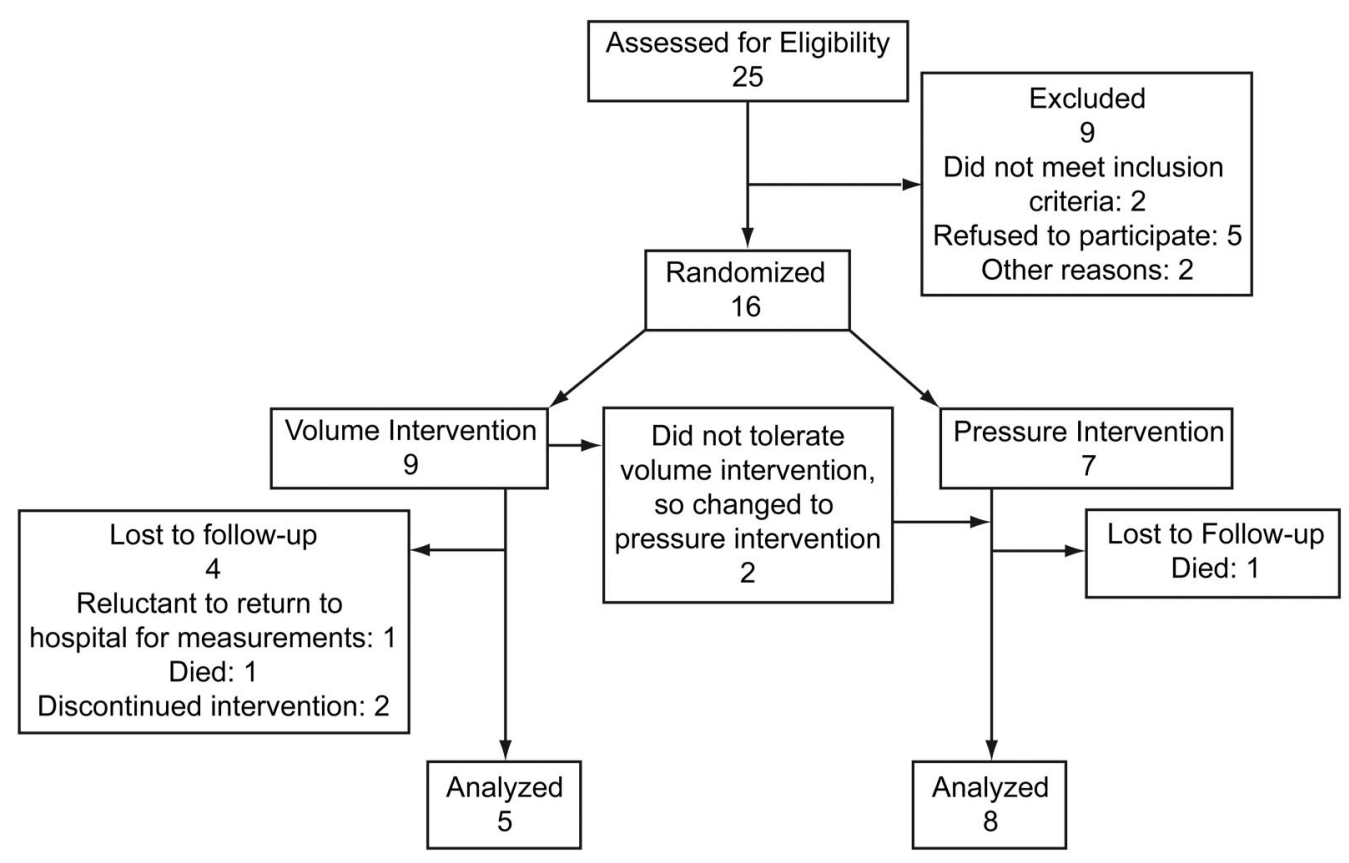

Fig. 1. Flow chart.

Table 1. Baseline Characteristics and Mean NIV Settings

\begin{tabular}{lcc}
\hline \hline & $\begin{array}{c}\text { Volume NIV } \\
(n=5)\end{array}$ & $\begin{array}{c}\text { Pressure NIV } \\
(n=8)\end{array}$ \\
\hline Male/female & $2 / 3$ & $6 / 2$ \\
Age (y) & $65 \pm 6$ & $67 \pm 9$ \\
Diagnosis, no. & & \\
$\quad$ Early-onset kyphoscoliosis & 2 & 4 \\
$\quad$ Post-polio & 3 & 2 \\
$\quad$ Post-rachitis & 0 & 1 \\
$\quad$ Thoracoplasty & $0.76 \pm 0.32$ & $0.84 \pm 0.20$ \\
FEV (L) & $1.10 \pm 0.36$ & $1.26 \pm 0.27$ \\
FVC (L) & $\mathrm{ND}$ & $21 \pm 3$ \\
Inspiratory pressure (cm $\left.\mathrm{H}_{2} \mathrm{O}\right)$ & $\mathrm{ND}$ & $6 \pm 3$ \\
Expiratory pressure $\left(\mathrm{cm} \mathrm{H}_{2} \mathrm{O}\right)$ & $0.64 \pm 0.13$ & $\mathrm{ND}$ \\
Tidal volume (L) & $21 \pm 2$ & $18 \pm 2$ \\
Respiratory rate (breaths/min) & & \\
& & \\
\hline values are mean \pm SD. & & \\
NIV = noninvasive ventilation & & \\
ND = no data (not measured) & & \\
\hline
\end{tabular}

nor significant change over time (Table 2). We also found improvement in $\mathrm{P}_{\mathrm{aO}}$ at discharge and at 3 months, compared to baseline, but the difference was significant only in the volume group, and there was no significant difference between the groups.

\section{Discussion}

There was no significant difference in days needed to establish NIV in a homogenous group of patients with chest-
Table 2. Mean $\mathrm{P}_{\mathrm{aCO}_{2}}$ and $\mathrm{P}_{\mathrm{aO}}$

\begin{tabular}{lcc}
\hline \hline & Volume NIV & Pressure NIV \\
\hline $\mathrm{P}_{\mathrm{aCO}_{2}}(\mathrm{~mm} \mathrm{Hg})$ & & \\
Baseline & $54 \pm 5$ & $57 \pm 8$ \\
At discharge & $45 \pm 7$ & $40 \pm 9^{*}$ \\
At 3 months & $40 \pm 10^{*}$ & $42 \pm 10^{*}$ \\
$\mathrm{P}_{\mathrm{aO}}(\mathrm{mm} \mathrm{Hg})$ & & \\
Baseline & $61 \pm 20$ & $69 \pm 23$ \\
At discharge & $81 \pm 14^{*}$ & $77 \pm 17$ \\
At 3 months & $84 \pm 22^{*}$ & $78 \pm 15$ \\
& & \\
\pm values are mean \pm SD. & & \\
* Significantly different than baseline. & \\
NIV = noninvasive ventilation & \\
\hline
\end{tabular}

wall deformity, so our hypothesis was rejected. Both volume NIV and pressure NIV normalized $\mathrm{P}_{\mathrm{aCO}_{2}}$ and $\mathrm{P}_{\mathrm{aO}}$, and these improvements were maintained after 3 months of home NIV.

Two of our patients switched from volume NIV to pressure NIV, which suggests that they preferred pressure NIV. The crossover study by Laserna et al ${ }^{12}$ supports that conclusion; they found that subjective response and tolerance were slightly better with bi-level positive airway pressure. Also, Windisch et al, ${ }^{13}$ who studied patients with COPD and chestwall disorders, found more gastrointestinal adverse effects in the volume-NIV group. Tsuboi et al ${ }^{11}$ compared different ventilation modes, and found that 6 of their 26 patients who started with a volume pre-set ventilator changed to a pressure pre-set ventilator, but did so several years after starting longterm NIV. This highlights the importance of long-term follow-up studies. 


\section{Volume Versus Pressure NiV in Patients With Chest-Wall Deformity}

One of the strengths of our study is the homogeneity of the cohort. Because we limited this study to patients with chest-wall deformity who were NIV-naïve, we can be more confident about conclusions about that specific group of patients. Earlier studies mostly compared more heterogeneous groups, with chronic respiratory failure due to COPD, neuromuscular disorders, or obesity hypoventilation syndrome. ${ }^{6,13}$ Also, some studies included patients already established on pressure ventilation, ${ }^{8}$ which increased the risk of bias from a preference for a known NIV mode.

Important factors in successful NIV establishment include the capabilities and experience of the clinicians. Our center has a team of 10 nurses that specialize in home mechanical ventilation, and they have a great deal of experience in selecting the correct patients, ventilator, ventilation settings, and mask fitting. All the nurses worked on the same ward for at least one week, so the patients did not see more than 2 different nurses during the week of NIV initiation, which, in our opinion, provides consistency in treatment. Also unique in this study is that we measured arterial blood gas values in the ICU, which allowed overnight titration of the NIV settings.

\section{Limitations}

Our sample size was small, so a larger study is needed to confirm our findings. A study with longer follow-up should measure health-related quality of life, adverse effects, and, as described in the pilot study by Crisafulli et al, ${ }^{10}$ objective and subjective sleep quality.

A previous study ${ }^{8}$ of pressure and volume NIV used a crossover design, which made it possible to establish identical volumes of delivered ventilation for the groups. We included only NIV-naïve patients, which made a crossover design impossible, because after starting NIV the patients would no longer be NIV-naïve. This meant that we could not establish identical volumes of delivered ventilation in the groups, so one could argue that the differences between the pressure and volume NIV groups might be attributed to different volumes of delivered ventilation.

\section{Conclusions}

Although pressure NIV has gained in popularity, our results suggest that either pressure or volume NIV offers effective ventilation and that there is no difference in the number of days needed for the patient to adjust to using pressure versus volume NIV. For clinical practice we therefore sug- gest using either pressure or volume NIV, and that clinician experience and expertise is crucial in the choice of ventilation type. The number of days needed to establish NIV is not a discriminative variable for deciding whether to start with pressure versus volume NIV in naïve patients with chest-wall deformity.

\section{REFERENCES}

1. Evans TW. International Consensus Conferences in Intensive Care Medicine: non-invasive positive pressure ventilation in acute respiratory failure. Organised jointly by the American Thoracic Society, the European Respiratory Society, the European Society of Intensive Care Medicine, and the Société de Réanimation de Langue Française, and approved by the ATS Board of Directors, December 2000. Intensive Care Med 2001;27(1):166-178.

2. Carroll N, Branthwaite MA. Control of nocturnal hypoventilation by nasal intermittent positive pressure ventilation. Thorax 1988;43(5): 349-353.

3. Ellis ER, Grunstein RR, Chan S, Bye PT, Sullivan CE. Noninvasive ventilatory support during sleep improves respiratory failure in kyphoscoliosis. Chest 1988;94(4):811-815.

4. Hill NS, Eveloff SE, Carlisle CC, Goff SG. Efficacy of nocturnal nasal ventilation in patients with restrictive thoracic disease. Am Rev Respir Dis 1992;145(2 Pt 1):365-3671.

5. Leger P, Bedicam JM, Cornette A, Reybet-Degat O, Langevin B, Polu JM, et al. Nasal intermittent positive pressure ventilation. Longterm follow-up in patients with severe chronic respiratory insufficiency. Chest 1994;105(1):100-105.

6. Schönhofer B, Sonneborn M, Haidl P, Böhrer H, Köhler D. Comparison of two different modes for noninvasive mechanical ventilation in chronic respiratory failure: volume versus pressure controlled device. Eur Respir J 1997;10(1):184-191.

7. Meecham Jones DJ, Wedzicha JA. Comparison of pressure and volume preset nasal ventilator systems in stable chronic respiratory failure. Eur Respir J 1993;6(7):1060-1064.

8. Tuggey JM, Elliott MW. Randomised crossover study of pressure and volume non-invasive ventilation in chest wall deformity. Thorax 2005;60(10):859-864.

9. Windisch W. Impact of home mechanical ventilation on health-related quality of life. Eur Respir J 2008;32(5):1328-1336.

10. Crisafulli E, Manni G, Kidonias M, Trianni L, Clini EM. Subjective sleep quality during average volume assured pressure support (AVAPS) ventilation in patients with hypercapnic COPD: a physiological pilot study. Lung 2009;187(5):299-305.

11. Tsuboi T, Oga T, Machida K, Chihara Y, Matsumoto H, Niimi A, et al. Importance of ventilator mode in long-term noninvasive positive pressure ventilation. Respir Med 2009;103(12):1854-1861.

12. Laserna E, Barrot E, Beiztegui A, Quintana E, Hernández A, Castillo J. [Non-invasive ventilation in kyphoscoliosis: a comparison of a volumetric ventilator and a BIPAP support pressure device]. Arch Bronconeumol 2003;39(1):13-18. Article in Spanish. Erratum in: Arch Broncoeumol 2003;39(4):191.

13. Windisch W, Storre JH, Sorichter S, Virchow JC Jr. Comparison of volume- and pressure-limited NPPV at night: a prospective randomized cross-over trial. Respir Med 2005;99(1):52-59.

This article is approved for Continuing Respiratory Care Education credit. For information and to obtain your CRCE

(free to AARC members) visit www.RCJournal.com

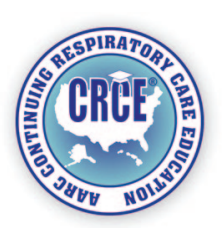

